\title{
Tracking of transplanted human umbilical cord-derived mesenchymal stem cells labeled with fluorescent probe in a mouse model of acute lung injury
}

\author{
GENGLONG LIU, HAIJIN LV, YULING AN, XUXIA WEI, XIAOMENG YI and HUIMIN YI \\ Surgical Intensive Care Unit, The Third Affiliated Hospital of Sun Yat-sen University, \\ Guangzhou, Guangdong 510630, P.R. China
}

Received June 18, 2017; Accepted January 18, 2018

DOI: $10.3892 /$ ijmm.2018.3491

\begin{abstract}
The aim of the present study was topreliminarily visualize the distribution of humanumbilical cord-derivedmesenchymal stem cells (hUC-MSCs) in treating acute lung injury (ALI) using a targeted fluorescent technique. Anovel fluorescent molecule probe was first synthesized via the specific binding of antigen and antibody in vitro to label the hUC-MSCs. Two groups of mice, comprising a normal saline (NS)+MSC group and lipopolysaccharide (LPS)+MSC group, were subjected to optical imaging. At $4 \mathrm{~h}$ following ALI mouse model construction, the labeled hUC-MSCs were transplanted into the mice in the NS+MSC group and LPS+MSC group by tail vein injection. The mice were sacrificed $30 \mathrm{~min}$, 1 day, 3 days and 7 days following injection of the labeled hUC-MSCs, and the lungs, heart, spleen, kidneys and liver were removed. The excised lungs, heart, spleen, kidneys and liver were then detected on asmall animal fluorescent imager. The fluorescent results showed that the signal intensity in the lungs of the LPS+MSC group was significantly higher, compared with that of the NS+MSC group at $30 \mathrm{~min}\left(3.53 \pm 0.06 \times 10^{-4}\right.$, vs. $1.95 \pm 0.05 \times 10^{-4}$ scaled counts/sec), 1 day $\left(36.20 \pm 0.77 \times 10^{-4}\right.$, vs. $23.45 \pm 0.43 \times 10^{-4}$ scaled counts/sec), 3 days $\left(11.83 \pm 0.26 \times 10^{-4}\right.$, vs.5.39 $\pm 0.10 \times 10^{-4}$ scaled counts/sec), and 7 days $\left(3.14 \pm 0.04 \times 10^{-4}\right.$,
\end{abstract}

Correspondence to: Professor Huimin Yi, Surgical Intensive Care Unit, The Third Affiliated Hospital of Sun Yat-sen University, 600 Tianhe Road, Tianhe, Guangzhou, Guangdong 510630, P.R. China

E-mail: ylhmin@hotmail.com

Abbreviations: hUC-MSCs, human umbilical cord-derived mesenchymal stem cells; ALI, acute lung injury; ARDS, acute respiratory distress syndrome; GD2, ganglioside; Cy7, CyDye mono-reactive NHS esters; LPS, lipopolysaccharide; DMEM, Dulbecco's modified Eagle's medium; PBS, phosphate-buffered saline; CCK-8, Cell Counting Kit-8; EBD, Evans blue dye; HE, hematoxylin and eosin; NS, normal saline; W/D, wet-to-dry ratio

Key words: fluorescent probe, visualization, mesenchymal stem cells, acute lung injury vs. $0.00 \pm 0.00 \times 10^{-4}$ scaled counts/sec; all $\left.\mathrm{P}<0.05\right)$. The fluorescence intensity in the liver of the LPS+MSC group, vs. $\mathrm{NS}+\mathrm{MSC}$ group was measured at $30 \mathrm{~min}\left(0.00 \pm 0.00 \times 10^{-4}\right.$, vs. $0.00 \pm 0.00 \times 10^{-4}$ scaled counts $\left./ \mathrm{sec}\right) ; 1$ day $\left(5.53 \pm 0.08 \times 10^{-4}\right.$, vs. $5.44 \pm 0.16 \times 10^{-4}$ scaled counts $\left./ \mathrm{sec}\right) ; 3$ days $\left(0.00 \pm 0.00 \times 10^{-4}\right.$, vs. $8.67 \pm 0.05 \times 10^{-4}$ scaled counts/sec); 7 days $\left(0.00 \pm 0.00 \times 10^{-4}\right.$, vs. $0.00 \pm 0.00 \times 10^{-4}$ scaled counts/sec). The signal intensity of the heart, spleen and kidneys was minimal. In conclusion, the novel targeted fluorescence molecular probe was suitable for tracking the distribution processes of hUC-MSCs in treating ALI.

\section{Introduction}

Acute lung injury (ALI), as an early and basic pathophysiologic change in acute respiratory distress syndrome (ARDS), is a significant contributor to morbidity and mortality rates in critically ill patients, and is most commonly caused by aspiration, infection, trauma and chemicals (1-3). The majority of current treatments for ARDS predominantly focus on restrictive intravenous fluid management and lung-protective ventilation, and there are currently few specific pharmacological therapies able to attenuate ALI and promote lung repair (4-6). ALI is a systemic inflammatory reaction syndrome, which is involved in a variety of inflammatory cells and inflammatory cytokines. Therefore, modulating immune responses and re-establishing the balance of the immune system is critical for the treatment of ALI (7).

Mesenchymal stem cells (MSCs) are one of the most promising adult stem cells, which have the ability of multiple differentiation potential for regenerative medicine, and can interact with the immune system in a complex and coordinate dmannerto induce a selective and balanced response (8). Current available evidence shows that MSCs are involved in the repair of the injured lung and effectively attenuateALI caused by various factors (9-11). Due to the uncertainty of their biological behavior, including distribution and chemotaxis in vivo following MSC transplantation, whether the use of MSCs in treating ALI is likely to be limited in their further application in clinical practice remains to be elucidated. Therefore, it is important to develop a suitable and noninvasive imaging probe to monitor the transplanted MSCs. 
In optical molecular imaging, fluorescence imaging utilizes a fluorescence molecular probe to mark specific molecules or cells, and its spatial resolution can reach the mm level (12). The probe has numerous advantages, including high sensitivity, high temporal resolution, low cost, and relatively high flux. In addition, it does not require any reaction substrates or other cofactors, and sensitive optical detection apparatus can directly track the space and time distribution of cells to understand its associated biological processes (13-15). Therefore, noninvasive fluorescence imaging techniques for the investigation of biological processes in small animal models are rapidly becoming an important tool in biomedical research. It has been successfully and diffusely applied in all fields, including life science, molecular biology and drug development $(16,17)$.

In the present study, monoclonal antibody anti-ganglioside (GD2) was used, which binds to the specific antigen GD2 expressed on hUC-MSCs as a carrier (18). It has been shown that cells selected by GD2 are a subpopulation of MSCs with the features of primitive precursor cells, and $\mathrm{Xu}$ et al (19) provided evidence that GD2 is a cell surface marker suitable for the isolation and purification of hUC-MSCs. A novel fluorescent molecule probe was first synthesized through the covalent coupling of anti-GD2 and fluorophore CyDye mono-reactive NHS esters (Cy7) (20). In the present study, according to the specific binding of antigen and antibody, synthetic anti-GD2-Cy7 wasincubated with hUC-MSCs in a standard culture medium (21). The labeled hUC-MSCs were transplanted into a lipopolysaccharide (LPS)-induced ALI mouse model to preliminarilyinvestigate the distribution processes of hUC-MSCs in treating ALI, which provides a theoretical basis for the establishment of an hUC-MSC treatment strategy for ALI in clinical work.

\section{Materials and methods}

Animal care. Healthy specific pathogen free male Balb/c mice (4-6 weeks old), purchased from Southern Medical University Experimental Animal Center (Guangzhou, China), were bred in the animal facility at the north campus of Sun Yat-sen University Laboratory Animal Center (Guangzhou, China) in a clean, temperature-controlled and independently ventilated environment, at $21^{\circ} \mathrm{C}$ under a $12 / 12 \mathrm{~h}$ dark/light cycle with ad libitum access to sterile water and standard pellet food under standardized environmental conditions. All experimental protocols were approved by the Institutional Animal Care and Use Committee of Sun Yat-sen University. Prior to commencing the experiments, all animals were subjected to a 2-week period of adaptation to the environment.

Materials. The Cy7 esters were purchased from Beijing Rich Encyclopedia Biological Technology Company (Beijing, China), The low-glucose Dulbecco's modified eagle medium (DMEM), fetal bovine serum and Dulbecco's phosphate-buffered saline (PBS) were purchased from Gibco; Thermo Fisher Scientific, Inc. (Waltham, MA, USA). The mouse anti-human GD2 monoclonal antibody (14.G2a; cat no. 554272) was purchased from BD Biosciences (San Jose, CA, USA)The Cell Counting Kit-8 (CCK-8) was purchased from Dojindo Molecular Technologies, Inc.(Kumamoto, Japan). The LPS (Escherichia coli; 0111:B4), Evans blue dye
(EBD) and trypsin were purchased from the Sigma-Aldrich; Merck Millipore (Darmstadt, Germany). Hematoxylin and eosin (HE) was obtained from the Pathology Department of The Third Affiliated Hospital of Sun Yat-sen University. hUC-USC isolation for experiments was performed by The Biological Treatment Center The Third Affiliated Hospital of Sun Yat-Sen University according to a protocol previously described (19). The obtained cryo preserved hUC-USCs were suitable for culture and were routinely passaged following thawing; apreliminary experiment confirmed that the hUC-USCs were capable of differentiating into osteogenic and adipogenic lineages (22).

Conjugation of antibody to fluorophore. The GD2-specific monoclonal antibody (anti-GD2) was labeled with the fluorophore $\mathrm{Cy} 7$ esters based on a previously described protocol $(23,24)$. Briefly, the monoclonal antibody was reconstituted at $1 \mathrm{mg} / \mathrm{ml}$ in $0.1 \mathrm{M}$ sodium bicarbonate; and $100 \mu \mathrm{l}$ was added to the reactive dye for conjugation. The antibody-dye mixtures were allowed to incubate for $1 \mathrm{~h}$ at room temperature, and then overnight at $4^{\circ} \mathrm{C}$. The conjugated antibody was then separated from the remaining un conjugated dye on a purification column by centrifugation for $5 \mathrm{~min}$ at $1,100 \mathrm{xg}$ and $4^{\circ} \mathrm{C}$.

Absorption spectrum and determining the degree of labeling. A small quantity of anti-GD2, Cy7-NHS and Cy7-GD2 was separately diluted with PBS (1:100) and transferred into a cuvette with a $1-\mathrm{cm}$ path-length. The absorbance of the final samples was measured in the ultraviolet (UV)-visible spectrophotometer (Beckman Coulter, Inc., Brea, CA, USA) and UV spectra (wavelength from 200 to $900 \mathrm{~nm}$ ). According to the Cy7-GD2 absorbance at $280 \mathrm{~nm}$ (A280) and $750 \mathrm{~nm}$ (A750), the concentration of protein was calculated in the sample: Protein concentration $(\mathrm{M})=[\mathrm{A} 280-(\mathrm{A} 750 \mathrm{x} 0.04)] \mathrm{x}$ dilution factor/203,000, where 203,000 is the molar extinction coefficient $(\varepsilon)$ in $\mathrm{cm}^{-1} \mathrm{M}^{-1}$ of a typical $\mathrm{IgG}$ at $280 \mathrm{~nm}$. The degree of labeling was also determined: Moles dye per mole protein $=\mathrm{A} 750 \mathrm{x}$ dilution factor/ $\varepsilon$ dye $\mathrm{x}$ protein concentration (M), where 250,000 is the approximate molar extinction coefficient of Cy7.

Cell labeling. The hUC-MSCs were seeded at a density of $5 \times 10^{5}$ cells/well on 24-well plates in $1 \mathrm{~mL}$ of complete DMEM and cultured for $24 \mathrm{~h}$ at $37^{\circ} \mathrm{C}$ in a fully humidified atmosphere of $5 \% \mathrm{CO}_{2}$. Cy7-GD2 was then directly added to the culture medium to label cells for $2 \mathrm{~h}$ in a standard culture medium at $37^{\circ} \mathrm{C}$ and $5 \% \mathrm{CO}_{2}$ by the specific binding of antigen and antibody. Following labeling, the medium was discarded, and the cells were washed three times with PBS to eliminate residual fluorescent-conjugated antibodies and resuspended in PBS. The labeled hUC-MSCs [ $1 \times 10^{6}$ cells in $200 \mu \mathrm{l}$ of normal saline (NS)], unlabeled hUC-MSCs $\left(1 \times 10^{6}\right.$ cells in $200 \mu \mathrm{l}$ of NS), $10 \mu \mathrm{l} \mathrm{Cy} 7-\mathrm{GD} 2$, and $20 \mu \mathrm{g} / \mathrm{ml} \mathrm{Cy} 7$ added to $200 \mu \mathrm{l}$ of NS were respectively collected in tubes and were detected on a small animal fluorescent imager.

In vitro cytotoxicity assay. A total of $5 \times 10^{3}$ hUC-MSCs or labeled hUC-MSCs were seeded per well with $100 \mu$ l culture medium in 96-well plates. The culture medium was carefully replaced following 1, 3, 12, 24 and $48 \mathrm{~h}$ of incubation 

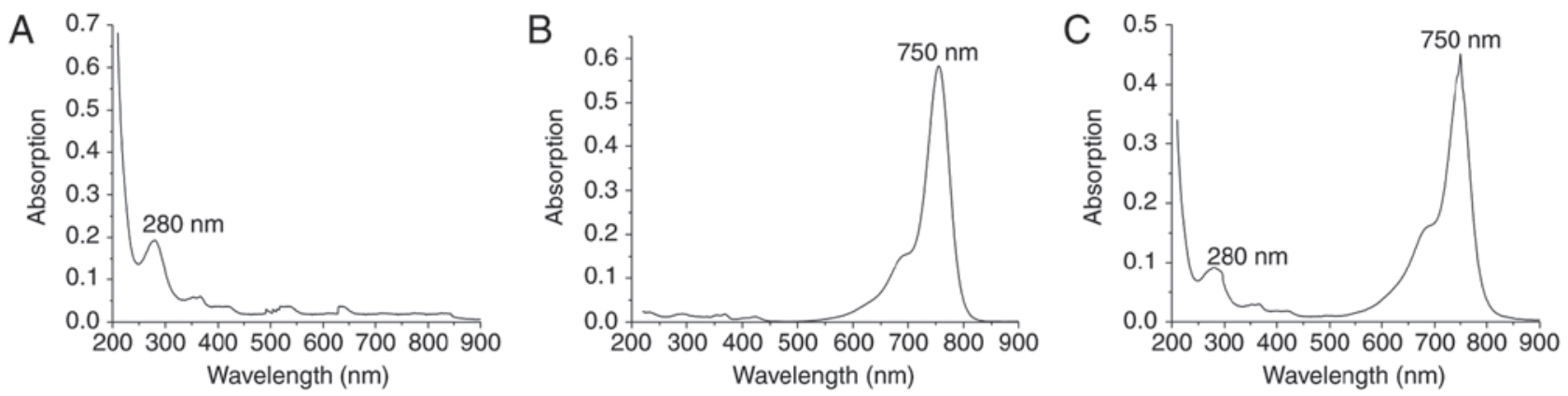

Figure 1. Absorption spectra of anti-GD2, Cy-NHS and Cy7-GD2. (A) Single maximum absorption peak of anti-GD2 at $280 \mathrm{~nm}$. (B) Single maximum absorption peak of Cy-NHS at $750 \mathrm{~nm}$. (C) Double absorption peaks of Cy7-GD2 at 280 and $750 \mathrm{~nm}$. GD2, ganglioside; Cy7, CyDye mono-reactive NHS esters.

at $37^{\circ} \mathrm{C}$ and $5 \% \mathrm{CO}_{2}$ with Cy7-GD2. CCK-8 (10 $\left.\mu \mathrm{l}\right)$ solution was added to each well and incubated for an additional $2 \mathrm{~h}$. The absorbance at $450 \mathrm{~nm}$ was measured on an Infinite F200 Multimode plate reader (Tecan Deutschland GmbH, Crailsheim, Germany). Cell viability was calculated by the labeled hUC-MSCs/unlabeled hUC-MSCs absorbance ratio.

Experimental design and LPS-induced lung injury. Briefly, the $\mathrm{Balb} / \mathrm{c}$ mice were randomly assigned into four groups: NS+NS group, NS+MSC group, LPS+NS group and LPS+MSC group ( $n=16$ per group). Following anesthetization of the mice with chloral hydrate $(10 \mathrm{mg} / \mathrm{kg})$, ALI was induced by the instillation of LPS (in the LPS+NS group and LPS+MSC group) from E. coli $\mathrm{O} 111: \mathrm{B} 4$ at $5 \mathrm{mg} / \mathrm{kg}$ intratracheally, whereas the mice in the NS+NS group and NS+MSC group were treated with sterile NS as controls (25). At $4 \mathrm{~h}$ post-LPS challenge, the mice in the NS+MSC group and LPS+MSC group were administered with hUC-MSCs $\left(1 \times 10^{6}\right.$ cells in $\left.200 \mu \mathrm{l} \mathrm{NS}\right)$ and those in the NS+NS group and LPS+NS group were administered with $200 \mu \mathrm{l}$ of NSvia injection into the tail vein. The animals were anesthetized and sacrificed by incision of the abdominal aorta at 24 and $48 \mathrm{~h}$ post-transplantation to harvest lung tissue samples.

Lung histopathology. The inferior lobes of the right lungs were cut into $5-\mu \mathrm{m}$ thick sections at each time point and subsequently stained with HE for histological analysis. Lung pathology was evaluated in a blinded-manner by a pathologist using a light microscope, according to four criteria: i) alveolar congestion, ii) hemorrhage, iii) infiltration oraggregation of neutrophils in the airspace or vessel wall, and iv) thickness of alveolar wall/hyaline membrane formation. Each category was graded on a $0-4$ point scale: 0 , no injury; 1 , injury up to $25 \%$ of the field; 2 , injury up to $50 \%$ of the field; 3 , injury up to $75 \%$ of the field; and 4 , diffuse injury. The total lung injury score was calculated as the sum of the four criteria (26).

Lung wet-to-dry (W/D) weight ratio. The superior and middle lobes of the right lungs were collected from the mice at each time point for assessment of the lung W/D ratio. The samples were weighed immediately following collection and then placed in a drying oven at $60^{\circ} \mathrm{C}$ for $72 \mathrm{~h}$, and the dry mass was subsequently determined. The wet lung mass was divided by the dry lung mass to give the W/D ratio.
Protein leakage from capillaries. Pulmonary micro vascular permeability was determined using the EBD extravasation method as described in a previous experiment (27). The EBD (20 mg/kg in $80 \mu \mathrm{l}$ PBS) was injected into the tail veinof the mice in every group at each time point. After $30 \mathrm{~min}$, the right ventricle of the heart was perfused with $10 \mathrm{ml}$ PBS to flush the lungs of the intravascular dye. Complete perfusion was confirmed when all blood had been cleared from the lung. The right lung tissue was then incubated in formamide for $48 \mathrm{~h}$ at $60^{\circ} \mathrm{C}$ and centrifuged for $30 \mathrm{~min}$ at $5,000 \mathrm{x} \mathrm{g}$ at $4^{\circ} \mathrm{C}$. The concentration of EBD in lung tissues was determined via spectrophotometric means at an optical density of $620 \mathrm{~nm}$.

Fluorescence imaging analyses. The NS+MSC group of mice $(n=12)$ and LPS+MSC group of mice $(n=12)$ were subjected to optical imaging. ALI was induced by instillation of LPS $(5 \mathrm{mg} / \mathrm{kg})$ intratracheally, as above. At $4 \mathrm{~h}$ post-LPS administration, the labeled hUC-MSCs were transplanted into mice in the NS+MSC group and LPS+MSC group by tail vein injection. The mice were sacrificed $30 \mathrm{~min}, 1$ day, 3 days and 7 days following the labeled hUC-MSC injection, and the lungs, livers, hearts and kidneys were removed. The excised lungs, livers, hearts and kidneys at each time point were imaged on a Maestro In-Vivo Optical Imaging system (excitation, 747 nm; emission, 774 nm; exposition time, 5,000 ms; Caliper Life Sciences, Woburn, MA, USA) (28). Images were captured and analyzed using Maestro 2.10.0 software (Caliper Life Sciences). The fluorescence intensity of each organ was measured by placing regions of interest (ROIs) on the organ and determined by an individual blinded to the experimental groups. The average signals were normalized to the exposure time and the area of the ROI (scaled counts/sec) (29).

Statistical analysis. Statistical analysis of the data was performed using one-way analysis of variance followed by Dunnett's post-hoc testor anun paired Student's t-test with SPSS software (version 17.0, SPSS, Inc., Chicago, IL, USA). All data are presented as the mean \pm standard deviation. $\mathrm{P}<0.05$ was considered to indicate a statistically significant difference. All statistical tests were two-sided.

\section{Results}

Absorption spectrum of Cy7-GD2. The absorption spectrum is the characteristic absorption curve of a material 

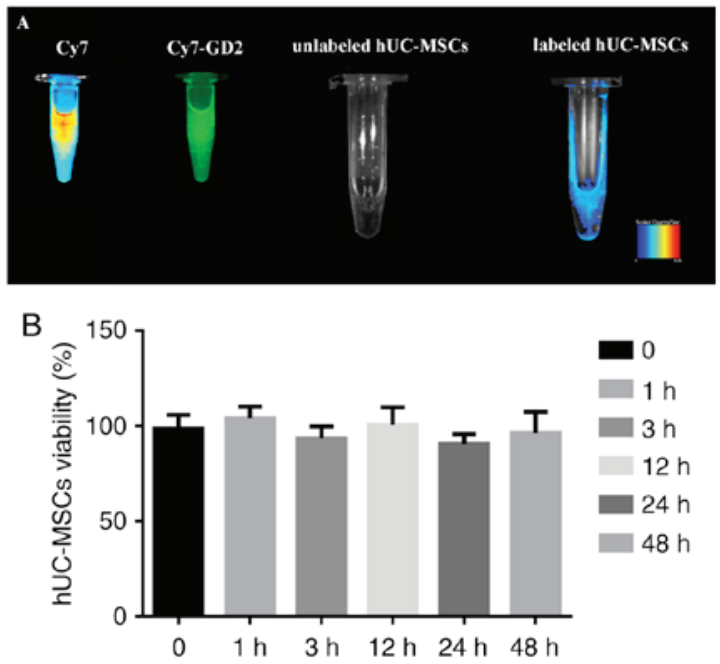

Figure 2. Cell fluorescence imaging and CCK-8 assay of the novel fluorescent molecule probe. (A) Labeled hUC-MSC suspension exhibited moderate fluorescence on small animal fluorescent imager, whereas none was detected in the unmarked hUC-MSC suspension. The hUC-MSCs were efficiently labeled by Cy7-GD. (B) CCK-8 assay to evaluate the cytotoxicity of the Cy7-GD2 complexes. No statistically significant difference was found between the two groups $(\mathrm{P}>0.05)$. The results are presented as the mean \pm standard deviation ( $\mathrm{n}=5$ per group). hUC-MSCs, human umbilical cord-derived mesenchymal stem cells; CCK-8, Cell Counting Kit-8; GD2, ganglioside; Cy7, CyDye mono-reactive NHS esters.

and is strictly consistent with molecular structure (30). The molecular structures of different materials have a unique spectrum. UV-Vis the absorption spectrum commonly used to examine the structure of a substance. There is a maximum absorption wavelength foranti-GD2 and Cy7-NHS, namely $280 \mathrm{~nm}$ (Fig. 1A) and $750 \mathrm{~nm}$ (Fig. 1B), respectively. The absorption spectrum of Cy7-GD2 has two peaks at 280 and $750 \mathrm{~nm}$ (Fig. 1C). The product Cy7-GD2 is able to maintain the properties of the monoclonal antibody and Cy7 dyes, which does not alter the structures or biological characteristics of these chemicals due to a conjugation reaction. According to A280 $=0.09143 ; \mathrm{A} 750=0.45129$ : Protein concentration $(\mathrm{M})=[\mathrm{A} 280-(\mathrm{A} 750 \times 0.04)] \times$ dilution factor $/ 20$ $3,000=(\mathrm{A} 280-0.04 \times \mathrm{A} 750) / 203,000=(0.09669-0.04 \times 0.41529) /$ $203,000=3.61 \times 10^{-7} \mathrm{M}$ Degree of label: Moles dye per mole protein $=\mathrm{A} 750 \times$ dilution factor/ $/$ dye $\times \mathrm{M}=\mathrm{F} / \mathrm{P}=\mathrm{A} 750 / 250,000$ x $\mathrm{M}=5.014$.

In vitro fluorescence imaging and CCK-8 assay. Based on the fluorescence imaging, the $\mathrm{Cy} 7$ reactive dye solution exhibited intense fluorescence; Cy7-GD2 emitted secondary fluorescence, the labeled hUC-MSC suspension exhibited moderate fluorescence, and the fluorescence signal of the unmarked hUC-MSC suspension was not fully detected (Fig. 2A). The CCK-8 assay confirmed that there was no significant difference in the cell viability of the experimental group and control group following extra incubation for 1, 3, 12, 24 and $48 \mathrm{~h}$ (Fig. 2B); it revealed that Cy7-GD2 had no cytotoxic effect on the hUC-MSCs.

ALI mouse model and assessing the validity of hUC-MSCs in treating ALI. The histological analysis indicated that LPS caused capillary expansion and congestion, in addition to neutrophil infiltration into the lung tissue. In addition, lung septa were noticeably thickened. The administration of
A

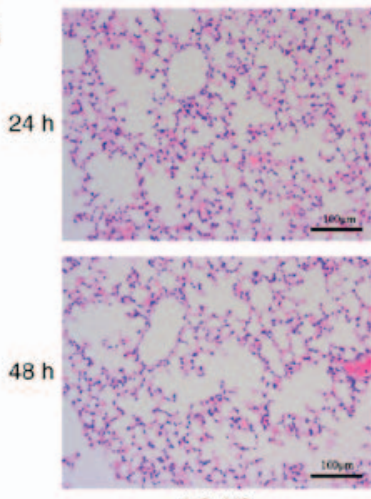

NS+NS

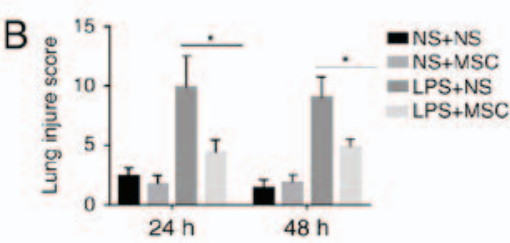

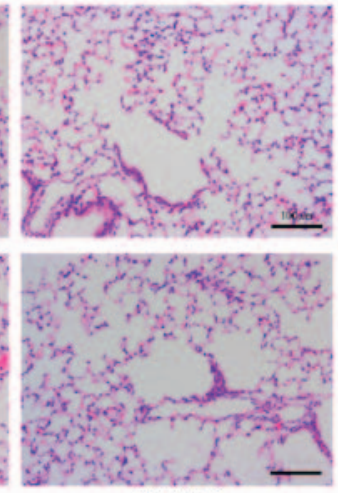

NS+MSC

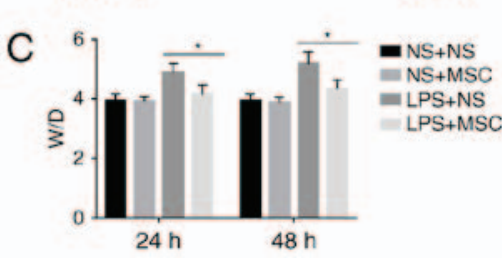

LPS+NS
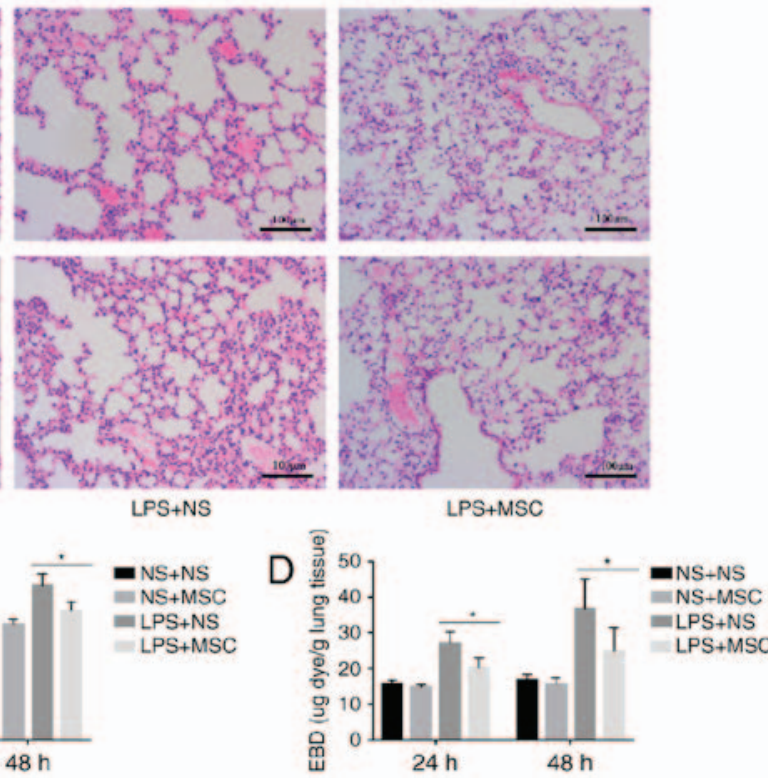

Figure 3. Effects of hUC-MSCs on LPS-induced acute lung injury. (A) HE staining (magnification, x200; scale bar=100 $\mu$ m) of lung sections from LPS-challenged mice at 24 and $48 \mathrm{~h}$ showed marked interalveolar septal thickening, extensive inflammatory infiltration, diffuse interstitial and alveolar edema, and severe interstitial hemorrhage. The administration of hUC-MSCs in the LPS+MSC group improved the lung histopathology to differing degrees. (B) At 24 and 48 h, HE staining of the lung sections from hUC-MSC-treated mice in the LPS+MSC group had significantly less injury, compared with mice in the LPS+NS group. (C) Mice administered with hUC-MSCs in the LPS+MSC group showed a trend towards a lower lung W/D weight ratio at 24 and $48 \mathrm{~h}$. (D) Dye accumulation in the lungs increased markedly at 24 and $48 \mathrm{~h}$ in the LPS+NS group, and was reduced by hUC-MSC treatment at 24 and 48 h. The results are expressed as the mean \pm standard deviation ( $\mathrm{n}=8$ per group). ${ }^{*} \mathrm{P}<0.05$. hUC-MSCs, human umbilical cord-derived mesenchymal stem cells; LPS, lipopolysaccharide; NS, normal saline; W/D, wet/dry ratio; EBD, Evans blue dye. 

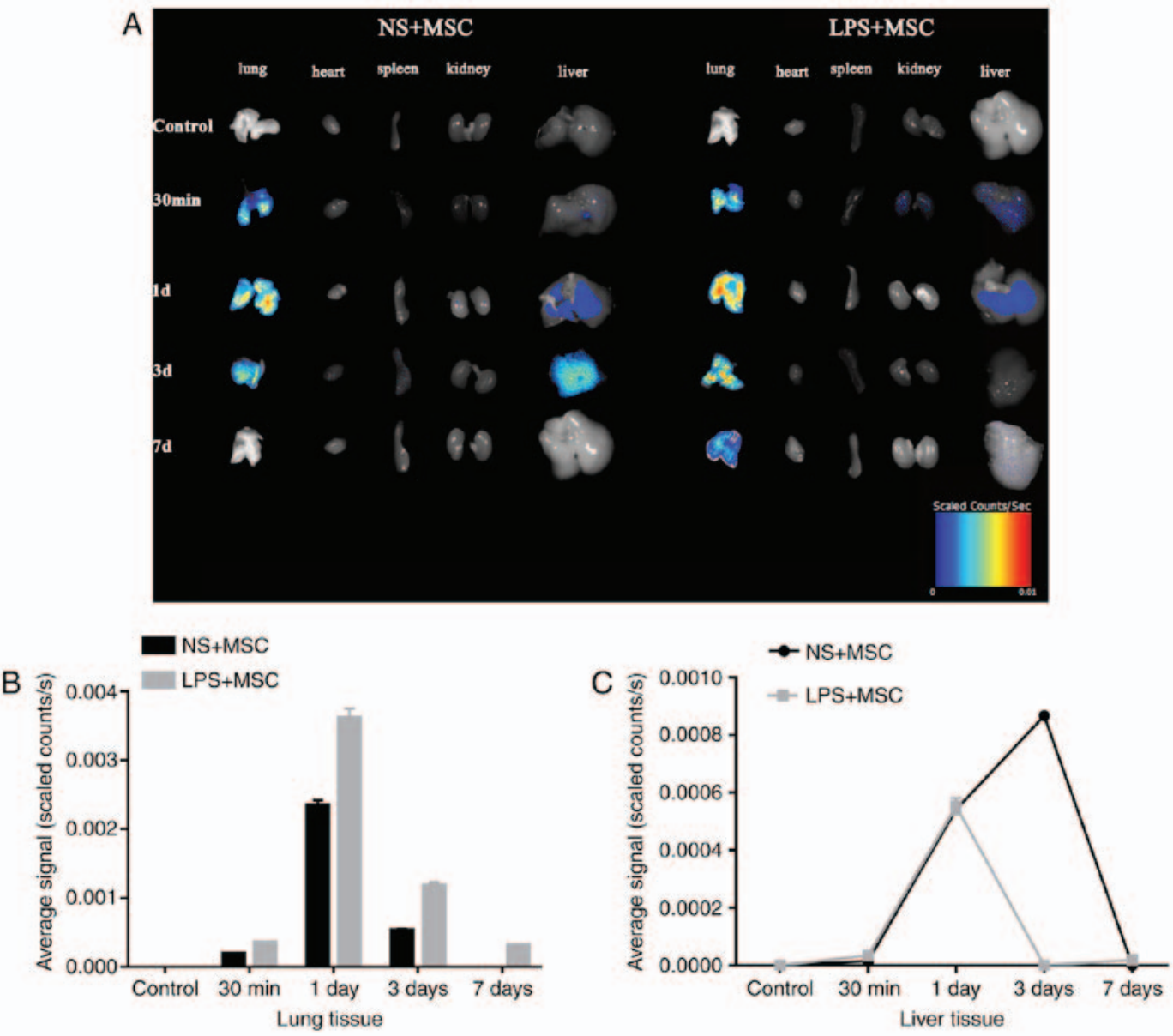

Figure 4. Distribution of fluorescence in organs, and fluorescence intensity of lungs and liver at each time point. (A) Fluorescent images of organs of mice at 30 min, 1 day, 3 days and 7 days following labeled hUC-MSC transplantation. (B) Average fluorescent signal intensity in the lung was quantified between 30 min and 7 days. (C) Quantified fluorescent signal intensity in the liver between 30 min and 7 days. The results are presented as the mean \pm standard deviation ( $n=3$ per group). hUC-MSCs, human umbilical cord-derived mesenchymal stem cells; LPS, lipopolysaccharide; NS, normal saline; d, day.

hUC-MSCs in the LPS+MSC group improved the lung injury at 24 and $48 \mathrm{~h}$ (Fig. 3A). Compared with the LPS+NS group, the lung tissue pathological score was relatively lower in the LPS+MSC group at 24 and $48 \mathrm{~h}$. However, there were no significant differences in the lung tissue pathological scores of the NS+MSC group and NS+NS group at the two time-points (Fig. 3B). In addition, the LPS+MSC group showed a trend toward a lower lung W/D ratio, compared with that in the LPS+NS group at 24 and $48 \mathrm{~h}$ (Fig. 3C). Compared with the LPS+NS group, the LPS+MSC group demonstrated improvement in pulmonary micro vascular permeability at 24 and $48 \mathrm{~h}$ (Fig. 3D).

Fluorescence imaging. The fluorescent tracing showed enhanced fluorescence signal in the lung $30 \mathrm{~min}$ following injection in the LPS+MSC group and NS+MSC group. The signal in the lungs of the LPS+MSC group and NS+MSC group peaked at day, with that of the LPS+MSC group decreasing gradually over time and detected 7 days following transplantation. However, the signal in the lungs of the NS+MSC group declined sharply and had disappeared completely at day 7. The fluorescence signal in the livers of the LPS+MSC group and NS+MSC group appeared at day 1, and that of the LPS+MSC group had almost disappeared at day 3. However, the fluorescence signal in the livers of the NS+MSC group enhanced gradually, peaking at day 3 and fading away at day 7 post-injection. The fluorescence signals of the heart, spleen and kidneys were minimal in the LPS+MSC group and NS+MSC group (Fig. 4A). The average fluorescence signal intensity of each organ was semi-quantitatively measured at each time point. The results showed that the signal intensity in the lungs of the LPS+MSC group was significantly higher, compared with that of the NS+MSC group at each time point: $30 \mathrm{~min}\left(3.53 \pm 0.06 \times 10^{-4}\right.$, vs. $1.95 \pm 0.05 \times 10^{-4}$ scaled counts/sec); 1 day $\left(36.20 \pm 0.77 \times 10^{-4}\right.$, vs. $23.45 \pm 0.43 \times 10^{-4}$ scaled counts/sec); 3 days $\left(11.83 \pm 0.26 \times 10^{-4}\right.$, vs. $5.39 \pm 0.10 \times 10^{-4}$ scaled counts/sec); 7 days $\left(3.14 \pm 0.04 \times 10^{-4}\right.$, vs. $0.00 \pm 0.00 \times 10^{-4}$ scaled counts/sec), as shown in Fig. 4B and Table I (all $\mathrm{P}<0.05)$. The fluorescence intensity in the liver of the LPS+MSC group, vs. NS+MSC group was as follows: $30 \mathrm{~min}\left(0.00 \pm 0.00 \times 10^{-4}\right.$, vs. $0.00 \pm 0.00 \times 10^{-4}$ scaled counts/sec); 1 day $\left(5.53 \pm 0.08 \times 10^{-4}\right.$, vs. $5.44 \pm 0.16 \times 10^{-4}$ scaled counts $\left./ \mathrm{sec}\right) ; 3$ days $\left(0.00 \pm 0.00 \times 10^{-4}\right.$, vs. $8.67 \pm 0.05 \times 10^{-4}$ scaled counts $\left./ \mathrm{sec}\right) ; 7$ days $\left(0.00 \pm 0.00 \times 10^{-4}\right.$, vs. $0.00 \pm 0.00 \times 10^{-4}$ scaled counts $\left./ \mathrm{sec}\right)$. Detection of the signal intensity of the heart, spleen and kidney was minimal (Fig. 4C).

\section{Discussion}

The present study aimed to develop a suitable and noninvasive imaging tool to track the bio distribution processes of 
Table I. Fluorescence intensity of the lungs.

\begin{tabular}{lcccc}
\hline Group & $30 \mathrm{~min}$ & 1 day & 3 days & 7 days \\
\hline LPS+MSC & $3.53 \pm 0.06^{\mathrm{a}}$ & $36.20 \pm 0.77^{\mathrm{a}}$ & $11.83 \pm 0.26^{\mathrm{a}}$ & $3.14 \pm 0.04^{\mathrm{a}}$ \\
NS+MSC & $1.95 \pm 0.04$ & $23.45 \pm 0.43$ & $5.39 \pm 0.10$ & $0.00 \pm 0.00$ \\
T-value & 19.90 & 14.45 & 22.79 & 77.69 \\
P-value & $<0.01$ & $<0.01$ & $<0.01$ & $<0.01$ \\
\hline
\end{tabular}

Fluorescence intensity data are shown as $\times 10^{-4}$ scaled counts $/ \mathrm{sec}$ and presented as the mean \pm standard deviation $(\mathrm{n}=3)$. ${ }^{\mathrm{a}} \mathrm{P}<0.001, \mathrm{compared}$ with the NS+MSC group at each time point. hUC-MSCs, human umbilical cord-derived mesenchymal stem cells; LPS, lipopolysaccharide; NS, normal saline.

hUC-MSCs in treating ALI. A novel class of fluorescence molecular probe was synthesized, which targeted and effectively labeled hUC-MSCs. It was hypothesized that, using this method, transplanted hUC-MSCs may be successfully monitored using this fluorescence molecular probe.

Stem cell transplantation is considered as a potential therapy to prevent or reverse the deleterious effects of various types of tissue injury and immunological disorders $(31,32)$. MSCs derived from human umbilical cord have the capacity to differentiate into various components of the hematopoietic niche, including bone and adipose under appropriate conditions (33). hUC-MSCs also possess high cell vitality, low immunogenicity, high paracrine potential and potent immune modulation to accelerate the repair process of injured tissue $(34,35)$. Animal models demonstrate that MSCs can induce the repair of injured organs and ameliorate inflammatory processes, including ALI/ARDS (36). The present study found that the infusion of hUC-MSCs had protective effects on attenuating lung injury and inhibiting lung inflammation in ALI mice induced by intratracheal LPS challenge. Lung histopathology and pathological scores, lung W/D ratio and pulmonary microvascular permeability were assessed to evaluate the effectiveness of hUC-MSCs in treating ALI, the results of which indicated that the lung histopathology and scores, pulmonary edema and permeability of lung microvasculature in the MSC+ALI group showed improvement, compared with those in the NS+ALI group, which were in accordance with a previous study that hUC-MSC administration attenuated lung inflammation in ALI, regardless of whether the cells were delivered at 24 or $48 \mathrm{~h}$ (37).

MSCs modified by different labeling strategies can be visualized using imaging systems, including magnetic resonance imaging (MRI), radionuclide imaging, including single photon emission computed tomography and positron emission tomography, and optical imaging, including fluorescence imaging and bioluminescence imaging (38). MRI appears to be the preferred option for imaging the distribution of magnetically-labeled stem cells due to its high spatial/temporal resolution and free selection of imaging plane (39). However, considering the principle of MRI and low $\mathrm{H}_{1}$ proton density of lung tissue (40), the efficacy of common MRI in monitoring the processes of hUC-MSCs in treating ALI is far from satisfactory. There exists radiation to viable cells in radionuclide imaging, which can lead to considerable toxicity to labeled cells and their normal organization, and can increase the risk of cancer (41). Another popular in vivo image tracing method is transfecting reported genes, including GFP, YFP or RFP, to express visible fluorescent proteins. The main advantage of this technique is that the fluorescence signal is stably expressed in offspring cells. However, gene editing increases the risk of viral infection and potentially carcinogenesis (42).

Fluorescence imaging offers the benefits of high contrast and sensitivity, low cost, ease of use, and safety, and is a suitable tracer method for the visualization of cells and tissues (43). Fluorescence imaging techniques have become an indispensable tool in biomedical investigations, as advances in photonic technology and noninvasive strategies have led to the widespread application of biological processes (44). In the present study, near-infrared $(650-900 \mathrm{~nm})$ fluorescent $\mathrm{Cy} 7$ dye was used for labeling several molecules for optical imaging due to its characteristics of small size, ideal aqueous solubility and a free amino structure (45). The Cy7-GD2 conjugate was synthesized by the coupling of Cy7-NHS with anti-GD2 consisting of N-terminal amines. The UV-vis spectrum showed that the complex had two distinctive peaks at 280 and $750 \mathrm{~nm}$ : The first peak was derived from anti-GD2 and the second peak was the characteristic peak originating from Cy7-NHS. No other molecule peaks were found, which not only contained the optical properties of Cy7, but also maintained the immune activity of monoclonal anti-GD2 antibody. This feature also provided the basis to calculate the F/Pratio of the conjugate, namely, the number of Cy7 units in the Cy7-GD2 conjugate. An F/P ratio of 5.014 is the ideal label status, with a critical 'optimum' at 4-9 labels/protein. Based on cell fluorescence imaging, the labeled hUC-MSCs suspension showed visible and moderate fluorescence. The results confirmed that the novel class of fluorescence molecular probe labeling hUC-MSCs was feasible. In addition, the complexes did not significantly affect the viability of the hUC-MSCs, demonstrating a low toxicity towards hUC-MSCs, which is essential for cell transfection.

To investigate the distribution of MSCs in a relevant ALI model, the hUC-MSCs labeled with fluorescent probe were administered into the ALI mice via tail vein injection. Multiple studies have demonstrated that the management of ALI/ARDS with MSCs involves two mechanisms (46). One of the mechanisms is termed the cell engraftment mechanism, during which transplanted MSCs can differentiate into type I and type II epithelial cells, fibroblasts and endothelial cells, subsequently leading to regeneration to repair the damaged 
tissue $(27,47)$. Another is the paracrine/endocrine mechanism, in which implanted MSCs secrete numerous and varied soluble mediators, including anti-inflammatory factors and growth factors, through paracrine/endocrine modes of action (48). However, the exact functions they exert in the repair of ALI remain to be fully elucidated, although the migration of MSCs to the diseased organs or tissues is required (49). The present study found that a host of hUC-MSCs migrated into the normal and injured lungs $30 \mathrm{~min}$ following cell engraftment, however, there was notable recruitment and increasing retention in the injured lungs at 1 day, extending to 7 days. These results indicated that hUC-MSCs possess the ability to gather in injured tissues, which is in accordance with previous reports that bone marrow-derived progenitor cells migrated to inflammatory areas of the lungs $(50,51)$. However, other studies have reported that there was only a low level of MSC retention in the injured lung $(52,53)$. The result of the present study revealed that the retention of hUC-MSCs in the LPS+MSC group was more marked, compared with that in the NS+MSC group at the same time between $30 \mathrm{~min}$ and 7 days post-cell transplantation. In particular, the localization of hUC-MSCs in the LPS+MSC group exhibited marked retention, whereas those in the NS+MSC group had completely disappeared on day 7 . Notably, it was also found that hUC-MSC recruitment to the liver appeared higher in the NS+MSC group, which suggested that a smaller proportion of hUC-MSCs accumulated in the injured lungs of the LPS+MSC mice, compared with that of the NS+MSC mice with the same MSC treatment strategy. Therefore, this discrepancy in benefits of hUC-MSCs between the two groups indicated that the persistence of transplanted hUC-MSCs in the lung was vital in promoting lung repair.

However, the mechanism underlying hUC-MSC migration and homing to the injured lung remains to be fully elucidated. Whether the injured lungs secrete several chemokines or whether the receptors and adhesion molecules for hUC-MSCs increase in the lungs to recruit hUC-MSCs to injured sites remains to be elucidated. To determine what types of homing cytokines are involved and how they function to recruit hUC-MSCs further investigation is warranted.

Taken together, the present study successfully constructed a novel targeted fluorescence molecular probe, Cy7-GD2, which not only contained the optical properties of $\mathrm{Cy} 7$, but also maintained the immune activity of anti-GD2 monoclonal antibodies. The hUC-MSCs also effectively alleviated the LPS-induced ALI in the mouse model. The hUC-MSCs labeled with fluorescent probe were also engrafted into the ALI mice to investigate the distribution of MSCs in the ALI mouse model. The novel fluorescent molecular probe, Cy7-GD2, was confirmed as a candidate for use to track the distribution of hUC-MSCs in treating ALI.

\section{Acknowledgements}

The authors would like to thank the Fundamental Research Funds of Science and Technology Planning Project of Guangdong Province, China (grant no. 411265834060) and the Key Scientific and Technological Program of Guangzhou City (grant no. 201508020262) for their support and the Biological Treatment Center The Third Affiliated Hospital of Sun Yat-Sen University for providing the hUC-MSCs.

\section{Competing interests}

The authors declare that they have no competing interests.

\section{References}

1. ARDS Definition Task Force; Ranieri VM, Rubenfeld GD, Thompson BT, Ferguson ND, Caldwell E, Fan E, Camporota L and Slutsky AS: Acute respiratory distress syndrome: The Berlin Definition. JAMA 307: 2526-2533, 2012.

2. Bellani G, Laffey JG, Pham T, Fan E, Brochard L, Esteban A, Gattinoni L, van Haren F, Larsson A, McAuley DF, et al: Epidemiology, patterns of care, and mortality for patients with acute respiratory distress syndrome in intensive care units in 50 countries. JAMA 315: 788-800, 2016.

3. Zafar K: Incidence of acute respiratory distress syndrome. JAMA 316: 347, 2016.

4. Papazian L, Forel JM, Gacouin A, Penot-Ragon C, Perrin G, Loundou A, Jaber S, Arnal JM, Perez D, Seghboyan JM, et al: Neuromuscular blockers in early acute respiratory distress syndrome. N Engl J Med 363: 1107-1116, 2010.

5. Sueblinvong V and Weiss DJ: Cell therapy approaches for lung diseases: Current status. Curr Opin Pharmacol 9: 268-273, 2009.

6. Briel M, Meade M, Mercat A, Brower RG, Talmor D, Walter SD, Slutsky AS, Pullenayegum E, Zhou Q, Cook D, et al: Higher vs. lower positive end-expiratory pressure in patients with acute lung injury and acute respiratory distress syndrome: Systematic review and meta-analysis. JAMA 303: 865-873, 2010.

7. Prockop DJ and Oh JY: Mesenchymal stem/stromal cells (MSCs): Role as guardians of inflammation. Mol Ther 20: 14-20, 2012.

8. Lv FJ, Tuan RS, Cheung KM and Leung VY: Concise review: The surface markers and identity of human mesenchymal stem cells. Stem Cells 32: 1408-1419, 2014.

9. Akram KM, Samad S, Spiteri M and Forsyth NR: Mesenchymal stem cell therapy and lung diseases. Adv Biochem Eng Biotechnol 130: 105-129, 2013.

10. Ho MS, Mei SH and Stewart DJ: The Immunomodulatory and therapeutic effects of mesenchymal stromal cells for acute lung injury and sepsis. J Cell Physiol 230: 2606-2617, 2015.

11. Shalaby SM, El-Shal AS, Abd-Allah SH, Selim AO, Selim SA, Gouda ZA, Abd El Motteleb DM, Zanfaly HE, El-Assar HM and Abdelazim S: Mesenchymal stromal cell injection protects against oxidative stress in Escherichia coli-induced acute lung injury in mice. Cytotherapy 16: 764-775, 2014.

12. Shim SH., Xia C, Zhong G, Babcock HP, Vaughan JC, Huang B, Wang X, Xu C, Bi GQ and Zhuang X: Super-resolution fluorescence imaging of organelles in live cells with photoswitchable membrane probes. Proc Natl Acad Sci USA 109: 13978-13983, 2012.

13. Chi C, Du Y, Ye J, Kou D, Qiu J, Wang J, Tian J and Chen X: Intraoperative imaging-guided cancer surgery: From current fluorescence molecular imaging methods to future multi-modality imaging technology. Theranostics 4: 1072-1084, 2014.

14. Hussain T and Nguyen QT: Molecular imaging for cancer diagnosis and surgery. Adv Drug Deliv Rev 66: 90-100, 2014.

15. Kim JC, Lee JL, Yoon YS, Alotaibi AM and Kim J: Utility of indocyanine-green fluorescent imaging during robot-assisted sphincter-saving surgery on rectal cancer patients. Int J Med Robot 12: 710-717, 2016.

16. Chi C, Zhang Q, Mao Y, Kou D, Qiu J, Ye J, Wang J, Wang Z, Du Y and Tian J: Increased precision of orthotopic and metastatic breast cancer surgery guided by matrix metalloproteinase-activatable near-infrared fluorescence probes. Sci Rep 5: 14197, 2015.

17. Sonn GA, Behesnilian AS, Jiang ZK, Zettlitz KA, Lepin EJ, Bentolila LA, Knowles SM, Lawrence D, Wu AM and Reiter RE: Fluorescent image-guided surgery with an anti-prostate stem cell antigen (PSCA) diabody enables targeted resection of mouse prostate cancer xenografts in real time. Clin Cancer Res 22: 1403-1412, 2016.

18. Martinez C, Hofmann TJ, Marino R, Dominici M and Horwitz EM: Human bone marrow mesenchymal stromal cells express the neural ganglioside GD2: A novel surface marker for the identification of MSCs. Blood 109: 4245-4248, 2007.

19. Xu J, Liao W, Gu D, Liang L, Liu M, Du W, Liu P, Zhang L, Lu S, Dong C, Zhou B and Han Z: Neural ganglioside GD2 identifies a subpopulation of mesenchymal stem cells in umbilical cord. Cell Physiol Biochem 23: 415-424, 2009. 
20. Jasmin, Torres AL, Nunes HM, Passipieri JA, Jelicks LA, Gasparetto EL, Spray DC, Campos de Carvalho AC and Mendez-Otero R: Optimized labeling of bone marrow mesenchymal cells with superparamagnetic iron oxide nanoparticles and in vivo visualization by magnetic resonance imaging. $\mathrm{J}$ Nanobiotechnology 9: 4, 2011.

21. Camacho X, Machado CL, García MF, Gambini JP, Banchero A, Fernández M, Oddone N, Bertolini Zanatta D, Rosal C, Buchpiguel CA, et al: Technetium-99m- or Cy7-Labeled Rituximab as an Imaging Agent for Non-Hodgkin Lymphoma. Oncology 92: 229-242, 2017.

22. Pan GZ, Yang Y, Zhang J, Liu W, Wang GY, Zhang YC, Yang Q, Zhai FX, Tai Y, Liu JR, et al: Bone marrow mesenchymal stem cells ameliorate hepatic ischemia/reperfusion injuries via inactivation of the MEK/ERK signaling pathway in rats. J Surg Res 178: 935-948, 2012

23. Metildi CA, Kaushal S, Luiken GA, Talamini MA, Hoffman RM and Bouvet M: Fluorescently labeled chimeric anti-CEA antibody improves detection and resection of human colon cancer in a patient-derived orthotopic xenograft (PDOX) nude mouse model. J Surg Oncol 109: 451-458, 2014.

24. Park JY, Hiroshima Y, Lee JY, Maawy AA, Hoffman RM and Bouvet M: MUC1 selectively targets human pancreatic cancer in orthotopic nude mouse models. PLoS One 10: e0122100, 2015.

25. Tai WL, Dong ZX, Zhang DD and Wang DH: Therapeutic effect of intravenous bone marrow-derived mesenchymal stem cell transplantation on early-stage LPS-induced acute lung injury in mice. Nan Fang Yi Ke Da Xue Xue Bao 32: 283-290, 2012 .

26. Li J, Li D, Liu X, Tang S and Wei F: Human umbilical cord mesenchymal stem cells reduce systemic inflammation and attenuate LPS-induced acute lung injury in rats. J Inflamm (Lond) 9: 33, 2012.

27. He H, Liu L, Chen Q, Liu A, Cai S, Yang Y, Lu X and Qiu H: Mesenchymal stem cells overexpressing angiotensin-converting enzyme 2 rescue lipopolysaccharide-induced lung injury. Cell Transplant 24: 1699-1715, 2015.

28. Zhang Y, Fan S, Yao Y, Ding J, Wang Y, Zhao Z, Liao L, Li P, Zang $\mathrm{F}$ and Teng GJ: In vivo, near-infrared imaging of fibrin deposition in thromboembolic stroke in mice. PLoS One 7 : e30262, 2012.

29. Wang XY, Ju S, Li C, Peng XG, Chen AF, Mao H and Teng GJ: Non-invasive imaging of endothelial progenitor cells in tumor neovascularization using a novel dual-modality paramagnetic/near-infrared fluorescence probe. PLoS One 7: e50575, 2012.

30. Yin J, Swartz TE, Zhang J, Patapoff TW, Chen B, Marhoul J, Shih N, Kabakoff B and Rahimi K: Validation of a spectral method for quantitative measurement of color in protein drug solutions. PDA J Pharm Sci Technol 70: 382-391, 2016.

31. Garbern JC and Lee RT: Cardiac stem cell therapy and the promise of heart regeneration. Cell Stem Cell 12: 689-698, 2013.

32. Yu DX, Marchetto MC and Gage FH: Therapeutic translation of iPSCs for treating neurological disease. Cell Stem Cell 12: 678-688, 2013

33. Soleimani M and Nadri S: A protocol for isolation and culture of mesenchymal stem cells from mouse bone marrow. Nat Protoc 4: 102-106, 2009.

34. Lee M, Jeong SY, Ha J, Kim M, Jin HJ, Kwon SJ, Chang JW, Choi SJ, Oh W, Yang YS, et al: Low immunogenicity of allogeneic human umbilical cord blood-derived mesenchymal stem cells in vitro and in vivo. Biochem Biophys Res Commun 446 983-989, 2014.

35. El Omar R, Beroud J, Stoltz JF, Menu P, Velot E and Decot V: Umbilical cord mesenchymal stem cells: The new gold standard for mesenchymal stem cell-based therapies? Tissue Eng Part B Rev 20: 523-544, 2014.
36. Maron-Gutierrez T, Silva JD, Asensi KD, Bakker-Abreu I, Shan Y, Diaz BL, Goldenberg RC, Mei SH, Stewart DJ, Morales MM et al: Effects of mesenchymal stem cell therapy on the time course of pulmonary remodeling depend on the etiology of lung injury in mice. Crit Care Med 41: e319-333, 2013.

37. Tong L, Zhou J, Rong L, Seeley EJ, Pan J, Zhu X, Liu J, Wang Q, Tang X, Qu J, et al: Fibroblast Growth Factor-10 (FGF-10) mobilizes lung-resident mesenchymal stem cells and protects against acute lung injury. Sci Rep 6: 21642, 2016.

38. Nguyen PK, Riegler J and Wu JC: Stem cell imaging: From bench to bedside. Cell Stem Cell 14: 431-444, 2014.

39. Wu C, Li J, Pang P, Liu J, Zhu K, Li D, Cheng D, Chen J, Shuai X and Shan H: Polymeric vector-mediated gene transfection of MSCs for dual bioluminescent and MRI tracking in vivo. Biomaterials 35: 8249-8260, 2014.

40. Triphan SM, Breuer FA, Gensler D, Kauczor HU and Jakob PM: Oxygen enhanced lung MRI by simultaneous measurement of $\mathrm{T} 1$ and T2 $*$ during free breathing using ultrashort TE. J Magn Reson Imaging 41: 1708-1714, 2015

41. Gu E, Chen WY, Gu J, Burridge P and Wu JC: Molecular imaging of stem cells: Tracking survival, biodistribution, tumorigenicity, and immunogenicity. Theranostics 2: 335-345, 2012.

42. Herberts CA, Kwa MS and Hermsen HP: Risk factors in the development of stem cell therapy. J Transl Med 9: 29, 2011.

43. Feng T, Ai X, Ong H and Zhao Y: Dual-responsive carbon dots for tumor extracellular microenvironment triggered targeting and enhanced anticancer drug delivery. ACS Appl Mater Interfaces 8: 18732-18740, 2016.

44. Landau MJ, Gould DJ and Patel KM: Advances in fluorescent-image guided surgery. Ann Transl Med 4: 392, 2016.

45. Lin X, Zhu H, Luo Z, Hong Y, Zhang H, Liu X, Ding H, Tian H and Yang Z: Near-infrared fluorescence imaging of non-Hodgkin's lymphoma CD20 expression using Cy7-conjugated obinutuzumab. Mol Imaging Biol 16: 877-887, 2014.

46. Wang YY, Li XZ and Wang LB: Therapeutic implications of mesenchymal stem cells in acute lung injury/acute respiratory distress syndrome. Stem Cell Res Ther 4: 45, 2013.

47. Zhao YD, Ohkawara H, Vogel SM, Malik AB and Zhao YY Bone marrow-derived progenitor cells prevent thrombin-induced increase in lung vascular permeability. Am J Physiol Lung Cell Mol Physiol 298: L36-L44, 2010.

48. Qin ZH, Xu JF, Qu JM, Zhang J, Sai Y, Chen CM, Wu L and Yu L: Intrapleural delivery of MSCs attenuates acute lung injury by paracrine/endocrine mechanism. J Cell Mol Med 16: 2745-2753, 2012

49. Sohni A and Verfaillie CM: Mesenchymal stem cells migration homing and tracking. Stem Cells Int 2013: 130763, 2013.

50. Yamada M, Kubo H, Kobayashi S, Ishizawa K, Numasaki M, Ueda S, Suzuki T and Sasaki H: Bone marrow-derived progenitor cells are important for lung repair after lipopolysaccharide-induced lung injury. J Immunol 172: 1266-1272, 2004.

51. Liu L, He H, Liu A, Xu J, Han J, Chen Q, Hu S, Xu X, Huang Y, Guo F, et al: Therapeutic effects of bone marrow-derived mesenchymal stem cells in models of pulmonary and extrapulmonary acute lung injury. Cell Transplant 24: 2629-2642, 2015.

52. Beckett T, Loi R, Prenovitz R, Poynter M, Goncz KK, Suratt BT and Weiss DJ: Acute lung injury with endotoxin or NO2 does not enhance development of airway epithelium from bone marrow. Mol Ther 12: 680-686, 2005.

53. Mei SH, McCarter SD, Deng Y, Parker CH, Liles WC and Stewart DJ: Prevention of LPS-induced acute lung injury in mice by mesenchymal stem cells overexpressing angiopoietin 1 . PLoS Med 4: e269, 2007.

This work is licensed under a Creative Commons Attribution-NonCommercial-NoDerivatives 4.0 International (CC BY-NC-ND 4.0) License. 